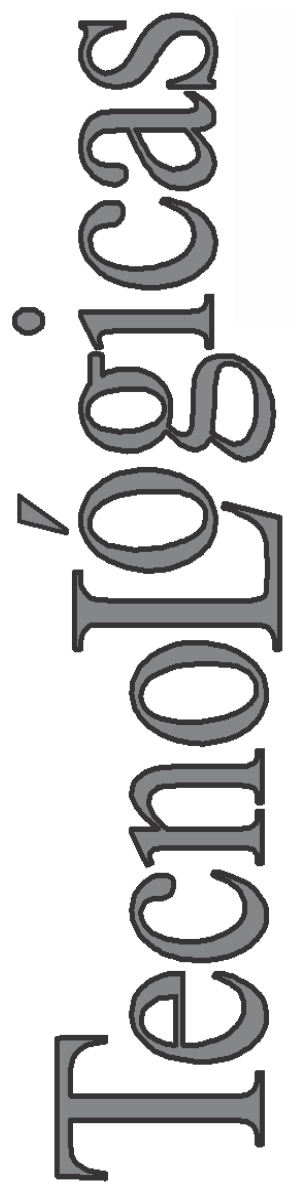

\title{
Introducción a la Visión Artificial mediante Prácticas de Laboratorio Diseñadas en Matlab
}

\section{Introduction to Artificial Vision through Laboratory Guides Using Matlab}

\author{
Verónica Londoño-Osorio ${ }^{1}$ \\ Jhovana Marín-Pineda ${ }^{2}$ \\ Eliana I. Arango-Zuluaga ${ }^{3}$
}

1 Facultad de Minas, Departamento de Energía Eléctrica y Automática, Universidad Nacional de Colombia Sede Medellín, Medellín-Colombia vlondonoo@unal.edu.co

2 Facultad de Minas, Departamento de Energía Eléctrica y Automática, Universidad Nacional de Colombia Sede Medellín, Medellín-Colombia jhmarinpi@unal.edu.co

3 Facultad de Minas, Departamento de Energía Eléctrica y Automática, Universidad Nacional de Colombia Sede Medellín, Medellín-Colombia eiarangoz@unal.edu.co 


\section{Resumen}

En este trabajo se presenta el diseño de dos prácticas de visión artificial para un curso que tiene el objetivo de introducir al estudiante en las diferentes áreas de profundización de su carrera. Por tanto, las prácticas diseñadas motivan y proporcionan contenidos adecuados al estudiante, además de incentivar la investigación en el área de procesamiento de imágenes. Se presenta una primera práctica introductoria, que explora los comandos básicos para el procesamiento de imagen mediante la programación de una interfaz gráfica de usuario en Matlab; y una segunda práctica en la que se utiliza un algoritmo de reconocimiento de imagen, que compara las características de color de imágenes faciales o de objetos. Se discuten los resultados, dificultades y recomendaciones para el desarrollo de cada sesión de práctica. Se muestran las respuestas a encuestas realizadas a los estudiantes para comprobar su nivel de aceptación del diseño y del contenido de las prácticas, así como su motivación para continuar profundizando en el área. Por último, se realizan comparaciones con prácticas diseñadas en otras universidades.

\section{Palabras clave}

Prácticas de laboratorio; procesamiento de imagen; visión artificial; interfaz gráfica de usuario (GUI); educación en ingeniería.

\section{Abstract}

This paper presents the design of two laboratory guides in artificial vision for a course which aims to introduce students to the different areas of specialization of his career. Therefore, the designed practices motivate and provide relevant content to the student, and to encourage research in the area of image processing. The first guide presents an introductory practice that explores the basic commands for image processing by programming a GUI in Matlab, and a second practice in which you use an image recognition algorithm, which compares the color characteristics of facial or objects images. The discussion of the results, challenges and recommendations for the development of each practice session are explained. The survey answers of the students are displayed. This survey allows checking their level of acceptance for the design and content of practice and motivation to continue studying in the image processing area. Finally, comparisons with laboratory guides that were designed in other universities are made.

\section{Keywords}

Laboratory guides; image processing; computer vision, graphical user interface (GUI); engineering education. 


\section{INTRODUCCIÓN}

Uno de los objetivos principales de la educación en la ingeniería es preparar a los estudiantes para la práctica profesional, de tal manera que estén capacitados para afrontar los retos que pueden presentarse en el ejercicio de su carrera. Además, en la aplicación práctica de los conceptos teóricos, pueden hallarse problemas para los cuales es necesario buscar alternativas de solución utilizando las competencias desarrolladas en el aula de clase. Por tanto un buen enfoque educativo debe incluir, además de la resolución de problemas teóricos que suelen despreciar muchas de las variables y obtener soluciones que funcionan sólo en la simulación, el planteamiento de aplicaciones reales que desarrollen en el alumno una mentalidad crítica y las habilidades para solucionar problemas prácticos (Feisel \& Rosa, 2005).

Otro de los objetivos de la educación superior actualmente es la formación de profesionales con capacidades y competencias para la investigación y la innovación, debido a su importancia para el crecimiento económico del país. Los espacios de práctica con recursos accesibles, que fomentan el descubrimiento autónomo y la transmisión de conocimiento, permiten alcanzar este objetivo (Gil \& Valdés, 1996).

Específicamente, en la temática de procesamiento de imágenes y visión artificial, muchos de los cursos contienen una gran carga teórica, dejando de lado la práctica que proporcionaría herramientas adecuadas para el diseño de aplicaciones. Por ejemplo, en el caso de la adquisición de imágenes pueden surgir perturbaciones reales, que no se observan en el trabajo con imágenes ideales, y afectan la capacidad de procesamiento de imágenes del algoritmo. Una perturbación puede ser un cambio de luz en el entorno, ante el cual es necesario tener elementos para capturar una imagen adecuada, esto es, tener experiencia en el uso de la cámara para capturar imágenes útiles para un algoritmo de visión artificial (Sebastián et al., 2003).

Otro ejemplo de la necesidad del trabajo práctico en los cursos de visión artificial, se encuentra en la elaboración de la base de datos que se utilizará para las comparaciones, ya que se deben tener en cuenta las fuentes de ruido, que podrían acarrear errores 
en los resultados de comparación entre las imágenes capturadas y las almacenadas en la base de datos (Gámez, 2009).

En el curso de Señales y sistemas lineales se hace necesario el diseño de prácticas introductorias a las diferentes áreas de la carrera, que motiven y proporcionen contenidos adecuados al estudiante para definir su área de profundización, además de incentivar la investigación. En el caso del área de procesamiento de imágenes y visión artificial, es necesaria la introducción del tema en prácticas de corta duración, que resuman el contenido teórico-práctico, que se quiere presentar al estudiante. Este tipo de prácticas no se han encontrado en la documentación consultada, en su lugar, se presentan cursos completos de un semestre de dedicación con sus respectivas prácticas de laboratorio (González, 2009; Sánchez, 2007).

El objetivo de este trabajo es presentar dos aplicaciones prácticas en visión artificial como herramienta de aprendizaje para el curso de Señales y sistemas lineales. En la siguiente sección se describe el contenido de las prácticas. La sección 3 presenta los resultados obtenidos al desarrollar las prácticas en clase y aplicar las encuestas a los estudiantes. La cuarta sección presenta una discusión de los resultados obtenidos y una comparación con trabajos de otros autores. Finalmente en la quinta sección se obtienen las conclusiones del trabajo realizado.

\section{DISEÑO dE LAS PRÁCTICAS DE LABORATORIO}

La elaboración de prácticas de laboratorio se inicia tomando en cuenta los recursos existentes en los laboratorios y su facilidad de acceso por parte de los estudiantes. Cada una de las prácticas contiene una parte inicial de fundamentación teórica, puesto que los alumnos deben adquirir conocimientos previos de cómo el programa procesa y descompone las imágenes, además de introducir algunos comandos básicos de Matlab. A continuación se describe el procedimiento que se sigue en cada una de las prácticas. 


\subsection{Práctica 1: Programación de una GUI (Interfaz Gráfica de Usuario)}

En el diseño de esta práctica se plantea el logro de los siguientes objetivos: Utilizar correctamente los comandos de procesamiento de imágenes de la herramienta Matlab, reconocer el método adecuado para procesar una imagen concreta dependiendo de la aplicación e implementar una GUI para el procesamiento de imágenes en Matlab. El enunciado comienza con un documento teórico que contiene los fundamentos sobre el tratamiento de imágenes en Matlab: Tipos de modelos de clasificación de las imágenes, forma de almacenamiento, Toolbox utilizados y ejemplos de comandos importantes.

Posteriormente, se le solicita al estudiante que realice la programación en Matlab de una GUI (Fig. 1) que permita las siguientes funciones: Capturar la imagen usando la cámara del computador, abrir y cerrar la imagen capturada y procesar la imagen según las funciones básicas solicitadas en el enunciado.

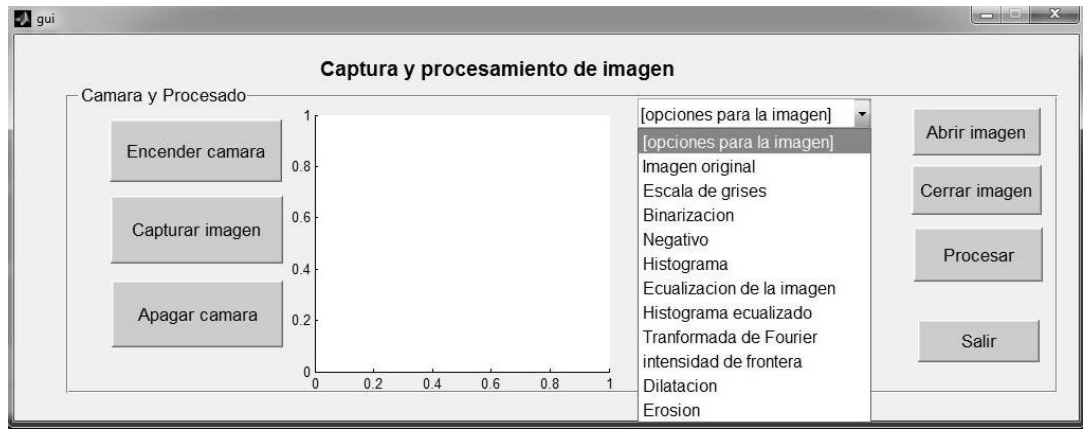

Fig. 1. Interfaz gráfica de usuario (GUI) propuesta a los estudiantes.

Fuente: Autores

Para realizar la captura de imágenes desde el Matlab el estudiante debe incluir los comandos en la GUI que le permitan activar la cámara, mostrar en una ventana gráfica la imagen previa a la captura, capturar la imagen y apagar la cámara. En el documento teórico se han incluido los comandos necesarios para obtener el formato de la cámara, el tamaño de la imagen (número de pixeles) y por tanto la resolución de la imagen. También se ha 
recomendado al estudiante realizar las capturas en un entorno suficientemente iluminado, con un fondo homogéneo y a la misma distancia; con el fin de evitar perturbaciones que pueden acarrear errores al realizar comparaciones. Para disminuir el efecto de las perturbaciones, existen filtros que ayudan a mejorar las imágenes y son útiles en caso de requerir un análisis más preciso.

Las funciones de procesamiento de imágenes que el estudiante debe implementar en su GUI, se pueden observar en la Fig. 1. Esto le permite conocer algunas funciones básicas que se aplican a las imágenes para obtener buena información acerca de lo que se va a procesar y buenos resultados en las comparaciones de algoritmos de visión artificial. Además, obtiene elementos para concluir que, los resultados de la aplicación de los comandos de procesamiento, son influenciados por factores como el entorno, el objeto de análisis, entre otros.

Por simplicidad en la programación de las funciones, cada una de ellas se aplicó en la imagen de intensidad o escala de grises y no en la imagen RGB (Formato Red-Green-Blue para las imágenes a color). Debido a que una imagen en formato RGB está formada por tres matrices, cada una con la información de las intensidades de cada color en el rango de 0 a 256. Por el contrario las imágenes de intensidad tienen solo una matriz con el mismo rango.

En la teoría inicial de la guía, se explica la forma aplicación de cada una de las funciones en la imagen capturada, se listan algunas sugerencias que permiten obtener mejores resultados y se presentan los ejemplos ilustrativos que se observan en la Fig. 2. Esta figura demuestra de izquierda a derecha y de arriba hacia abajo, los resultados al aplicar algunas de las funciones de procesamiento de imágenes: Escala de grises, binarización, negativo, dilatación, histograma, ecualización de la imagen, transformada de Fourier y erosión.

\subsection{Práctica 2: Aplicación de Visión Artificial para Seleccionar Objetos}

Los sistemas de visión artificial de la industria están orientados a realizar tareas de inspección, clasificación, control de calidad y control de procesos. Estos sistemas utilizan el procesamiento de 
imágenes para detectar las características de interés de los objetos, tales como: Defectos, dimensiones, formas y colores. En esta práctica se plantea una aplicación a nivel industrial de clasificación de objetos por comparación de colores, con uso del modelo RGB, para pasar de la teoría de visión artificial, a la aplicación, integrando la captura de imágenes.

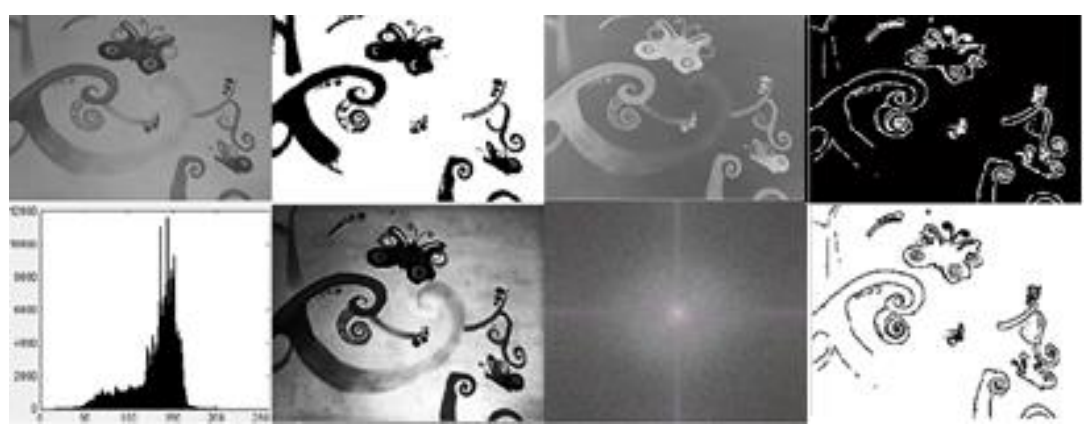

Fig. 2. Resultados de las funciones propuestas en la primera práctica.

Fuente: Autores

En el diseño de la práctica se ha tenido en cuenta que alumno desarrolle habilidades para: Realizar un programa de clasificación de objetos mediante el uso de los comandos de la primera práctica, analizar las tres capas del modelo RGB y ejemplificar el uso de la visión artificial.

La primera tarea que el estudiante debe ejecutar es la captura de las imágenes de la base de datos, aplicando lo desarrollado en la primera práctica. La base de datos se usa para comparar con las imágenes capturadas y debe contener imágenes de objetos de la misma clase y tamaño que los de la imagen a identificar, pero de diferentes colores. Para evitar las perturbaciones, se recomienda utilizar iluminación homogénea, fondo claro y la misma distancia de captura.

La segunda tarea es la realización del programa que captura la imagen a clasificar y la compara con la base de datos, usando un algoritmo basado en el modelo RGB para obtener el resultado de clasificación. El algoritmo propuesto facilita el entendimiento de las técnicas de visión artificial en esta práctica introductoria. 
El programa debe comenzar por la descomposición de las imágenes del modelo RGB en las tres matrices (Red, Green, Blue) que lo componen. A continuación se halla la sumatoria de los valores de intensidad en las columnas y filas de cada matriz, obteniendo un valor para cada color. Luego se realiza la comparación entre los valores de cada color de la imagen capturada y los de las imágenes de la base de datos, teniendo en cuenta un rango de tolerancia que se define según el criterio de selección del objeto, la importancia de los colores y el tamaño. Si los valores de la imagen capturada se encuentran dentro del rango de tolerancia establecido, en relación con los valores de las imágenes de la base de datos, se clasifica como un objeto permitido.

En la Fig. 3 se observa un ejemplo de imágenes de la base de datos que contiene las imágenes de un plátano verde y uno maduro, y la imagen a comparar, que para este caso en un plátano maduro. Además se muestran las dos posibles respuestas en cuadros de avisos del programa ante la comparación de una imagen capturada.
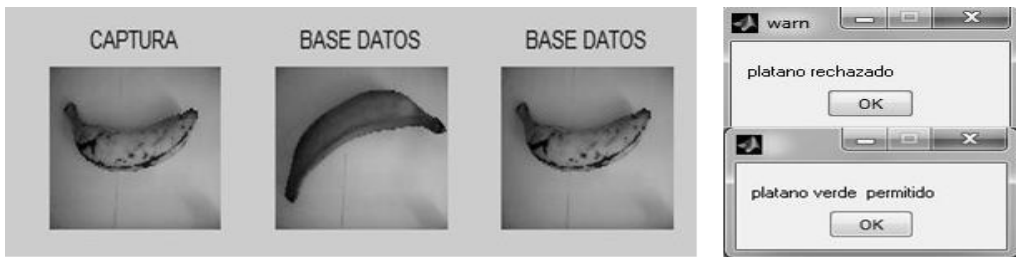

Fig. 3. Imagen capturada comparada con las imágenes en la base de datos y posibles respuestas del programa ante una comparación. Fuente: Autores

\section{RESULtAdOS DE LA APLICACIÓN DE LAS PRÁCTICAS}

Las prácticas se realizaron en el curso de Señales y sistemas lineales, del programa en Ingeniería de Control en la Universidad Nacional de Colombia - Sede Medellín. En general se observó una gran motivación por parte de los estudiantes para el aprendizaje de las técnicas de visión artificial usando los comandos de Matlab ya que se mostraron propositivos acerca del tema, completando en muchos casos lo solicitado con sus propias ideas y entregando buenos resultados de la implementación de cada práctica. 
En el desarrollo de la primera práctica se observó que para su desarrollo adecuado son necesarios los conocimientos previos de elaboración de una GUI en Matlab que permiten diseñar la GUI que se está solicitando. También se pudo observar que los ejemplos ilustrados del documento teórico facilitan la realización de la práctica a los estudiantes.

En la segunda práctica, es fundamental entender la combinación de matrices que hacen un color para restringir adecuadamente los rangos de tolerancia en la comparación de las imágenes. En general, los estudiantes implementaron el algoritmo de comparación propuesto y obtuvieron los resultados esperados aunque con algunas dificultades debido a que el algoritmo de comparación empleado no aísla las perturbaciones en la adquisición de las imágenes, tales como: luz, posición de la cámara, fondo, entre otras. Para mejorar los resultados se recomienda hacer uso de filtros específicos dependiendo de la aplicación. Otra observación obtenida del desarrollo de las prácticas, es recomendar a los estudiantes la utilización de estructuras de programación sin condicionales anidados para garantizar una comparación completa entre los valores para cada color.

Adicionalmente, para obtener mayor precisión y disminuir la sensibilidad ante perturbaciones, se presentan ejemplos a los estudiantes en los que se han considerado otras características de las imágenes del objeto a comparar, como por ejemplo, el tamaño, centro de masa, área, perímetro; el objetivo de esta información es fomentar el interés para profundizar en el tema.

Con el fin de retroalimentar la experiencia de los estudiantes con las prácticas y conocer sus opiniones acerca de la realización de las prácticas de visión artificial en el curso de Señales y sistemas lineales, se hizo una encuesta con 4 preguntas y un espacio abierto para sugerencias y observaciones.

Las preguntas realizadas y sus respuestas en forma gráfica, se pueden observar en la Fig. 4. En general, los resultados de las encuestas se valoran como muy positivos, los estudiantes reflejan una gran motivación e interés por el tema, además en los comentarios abiertos, resaltan como aspecto positivo el uso de herramientas que se encuentran a su alcance para dicho proceso, como por ejemplo el uso de las cámaras web de sus propios computado- 
res en la captura de imágenes, lo cual fue posible debido al objetivo introductorio de las mismas. También resaltan el hecho de que es se logra incentivar el interés en esta área de la carrera, debido a que se realizó un acercamiento práctico.

1. En una escala de 1 a 5 , valore su capacidad actual para elaborar un código de procesamiento de imagen con los comandos aprendidos.

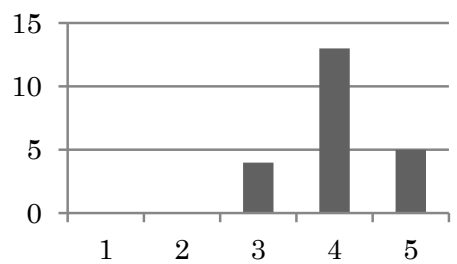

3. ¿La realización de las prácticas de visión artificial cumplió con sus objetivos?
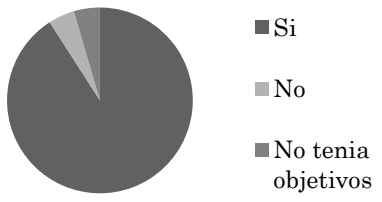

2. En una escala de 1 a 5 que tan adecuado considera usted incluir prácticas de visión artificialen la asignatura Señales y Sistemas lineales

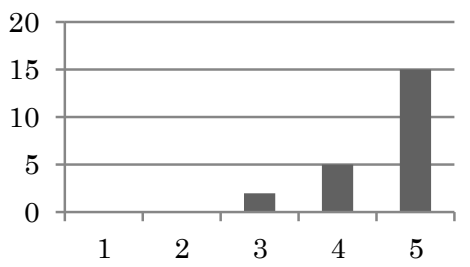

4. ¿Cómo valoraría su nivel de motivación para continuar profundizando en visión artificial o procesamiento de señales después de la elaboración de las prácticas?,

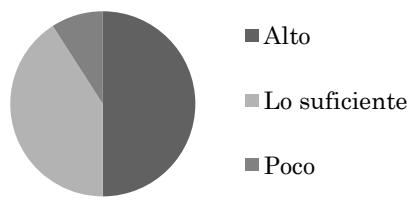

Fig. 4. Resultados gráficos de las encuestas. Fuente: Autores

\section{ANÁLISIS Y COMPARACIONES}

Las dos prácticas presentadas en este trabajo ofrecen una introducción general al desarrollo de aplicaciones en visión artificial con Matlab, debido a que se utiliza una metodología que permite al estudiante adquirir con rapidez el manejo de los comandos y métodos básicos. Este diseño de prácticas, contrasta con las que se pueden encontrar en la web, ya que al ser diseñadas para una asignatura completa de visión artificial, cada práctica detalla un 
tema específico visto en clase y no presentan una visión tan general del tema (González, 2009; Sánchez, 2007). Como se planteó inicialmente, el objetivo de las prácticas diseñadas es introductorio y motivacional, dirigido a los estudiantes de un curso que ofrece una visión general de las diferentes áreas de la carrera, con el fin de promover su futura profundización en los temas estudiados. Como se observa en los resultados de las encuestas, se logró motivar a los estudiantes para profundizar en el área.

$\mathrm{Al}$ realizar las comparaciones con las prácticas elaboradas para cursos de visión artificial en otras universidades (González, 2009; Sánchez, 2007; Fernández et al., 2005), se observaron algunas ventajas específicas que se detallan a continuación. Los ejemplos ilustrativos permiten al estudiante adquirir los elementos para implementar sus propios códigos en Matlab. La teoría necesaria para la realización de la práctica es la parte inicial de la guía; contiene el significado de los parámetros y los resultados de los comandos realizados, con el objetivo de facilitar las comparaciones y orientar a los alumnos; esto facilita la obtención de resultados por parte de los estudiantes y permite que la guía sea una referencia que se puede consultar para el desarrollo de futuras aplicaciones.

Otra de las diferencias, es que las guías diseñadas contienen las imágenes de cada paso, lo que facilita el entendimiento de la función de cada uno de los comandos. Por último, al diseñar la guía de laboratorio para ser resuelta con recursos de fácil acceso a los estudiantes, se facilita su implementación y se motiva la profundización del estudiante en el área de trabajo. Algunas de las prácticas de la bibliografía, usan equipos especializados que no están disponibles para los estudiantes y pueden desmotivar la realización de futuros proyectos en el área (Fernández et al., 2005).

\section{CONCLUSIONES}

Durante la elaboración e implementación de cada una de las prácticas se pudo observar que los estudiantes mejoran sus habilidades propositivas y argumentativas ya que se ven enfrentados 
con sistemas reales que requieren de su análisis y capacidad de solución de problemas.

Según se evidencia en las encuestas realizadas, el trabajo práctico en visión artificial por medio de las guías diseñadas, proporciona una apropiación del conocimiento, promueve el interés por la profundización en el tema y por la investigación. Además se resalta como aspecto positivo el uso de herramientas que se encuentran al alcance de los estudiantes. Las guías ofrecen una aproximación sencilla a la visión artificial, a través del algoritmo para reconocer objetos, el cual permite identificar los fundamentos que permitirán al estudiante continuar su profundización para desarrollos posteriores de aplicación industrial.

El desarrollo de las prácticas permitió comprobar a los estudiantes que cuando se trabaja con visión artificial es muy importante considerar las perturbaciones que se presentan en el medio ya que ellas pueden afectar en gran medida los resultados. Una de las principales dificultades que se observó, es la selección adecuada del entorno de captura de las imágenes, con luz homogénea y fondo claro que permita elaborar una correcta base de datos.

La comparación por colores se puede realizar con cualquier objeto y es una aplicación para demostrar una forma de seleccionar objetos. A nivel industrial se utilizan diversos análisis de color, tamaño y forma, usando filtros específicos de procesado de imágenes debido a que se requiere más precisión en el objeto identificado. En el caso de la práctica diseñada, el uso del algoritmo sencillo está justificado, ya que el objetivo es introducir al estudiante en el manejo de la visión artificial.

\section{AGRADECIMIENTOS}

Este trabajo ha sido desarrollado dentro del Grupo de Automática de la Universidad Nacional - GAUNAL, en la línea de investigación en Modelado y control de fuentes de potencia eléctrica, bajo el proyecto MPPT Vectorial. 


\section{REFERENCIAS}

Feisel, L.D., \& Rosa, A. J. (2005). The Role of the Laboratory in Undergraduate Engineering Education. Rev. Journal of Engineering Education, 94(1), 121-130.

Fernández C., Neco R. \& Asunción M. (2005). Webcams como equipo de prácticas en asignaturas de visión artificial y reconocimiento de patrones. En Congreso Internacional Virtual de Educación Universidad Miguel Hernández, Elche (Alicante), España.

Gámez, C. (2009). Diseño y desarrollo de un sistema de reconocimiento de caras. Universidad Carlos III de Madrid, Proyecto de fin de carrera.

Gil, D. \& Valdés, P. (1996). La orientación de las prácticas de laboratorio como investigación: un ejemplo ilustrativo. Rev. Enseñanza de las Ciencias. 14 (2), 155-163.

González, Y. (2009). Material de prácticas de laboratorio para el curso de Visión Industrial, Departamento de Matemáticas e Informática, Universidad de las Islas Baleares, Recuperado el 2 de julio de 2013, de http://dmi.uib.es/ ygonzalez/VI/Material_del_Curso/ Contenido_Practico.html

Sánchez, A. (2007). Prácticas de laboratorio para el curso de Visión Artificial de Ingeniería informática, Escuela Superior de Ciencias Experimentales y Tecnología, Universidad Rey Juan Carlos, Recuperado el 2 de julio de 2013, de http://www.escet.urjc.es/ visiona /ejercicios.html

Sebastián, J.M., García, D. \& Sánchez, F.M. (2003). Remote Access Education Based on Image Acquisition and Processing through the Internet”. IEEE Transactions on Education, 46, 142-148. 